\title{
An Alternative Q Chart Incorporating A Robust Estimator OfScale
}

Michael B. C. Khoo

Universiti Sains, Malaysia, mkbc@usm.my

Follow this and additional works at: http://digitalcommons.wayne.edu/jmasm

Part of the Applied Statistics Commons, Social and Behavioral Sciences Commons, and the Statistical Theory Commons

\section{Recommended Citation}

Khoo, Michael B. C. (2004) "An Alternative Q Chart Incorporating A Robust Estimator Of Scale," Journal of Modern Applied Statistical Methods: Vol. 3 : Iss. 1 , Article 9.

DOI: $10.22237 /$ jmasm/1083370140

Available at: http://digitalcommons.wayne.edu/jmasm/vol3/iss1/9

This Regular Article is brought to you for free and open access by the Open Access Journals at DigitalCommons@WayneState. It has been accepted for inclusion in Journal of Modern Applied Statistical Methods by an authorized editor of DigitalCommons@WayneState. 


\title{
An Alternative $Q$ Chart Incorporating A Robust Estimator Of Scale
}

\author{
Michael B.C. Khoo \\ School of Mathematical Sciences \\ Universiti Sains Malaysia
}

In overcoming the shortcomings of the classical control charts in a short runs production, Quesenberry (1991 \& 1995a - d) proposed $Q$ charts for attributes and variables data. An approach to enhance the performance of a variable $Q$ chart based on individual measurements using a robust estimator of scale is proposed. Monte carlo simulations are conducted to show that the proposed robust $Q$ chart is superior to the present $Q$ chart.

Key words: short runs; $Q$ chart; $Q_{\mathrm{MSSD}}$ chart; in-control; out-of-control (o.o.c.)

\section{Introduction}

Short runs production or more commonly known as short runs is given more emphasis in manufacturing industries nowadays. The trend which is emphasized now is low volume production. This trend is a result of extra emphasis on just-in-time (JIT) techniques, job shop settings and synchronous manufacturing. Classical SPC charting methods such as $\bar{X}, R$ and $S$ charts which assume high volume manufacturing processes require at least 25 or 30 calibration samples of size 4 or 5 each to be available in the estimation of the process parameters before on-line charting begins.

In a short runs production, there is often a paucity of relevant data available for estimating the process parameters and establishing control limits prior to a production run. It is desirable to begin charting at or very near the beginning of the run in these cases.

Michael B. C. Khoo is a lecturer at the University of Science of Malaysia. His research interests are statistical process control and reliability analysis. He is a member of the editorial board of Quality Engineering.
In a short runs environment, the process mean, $\mu$, and variance, $\sigma^{2}$, cannot be known before the production run is begun because they change from run to run. For the individual measurements situation based on variables data, Quesenberry (1991 \& 1995a) proposed the following four statistics for cases where $\mu$ and $\sigma^{2}$ are known and unknown. The notations in Table 1 are used:

1. Case KK: $\mu=\mu_{0}, \sigma=\sigma_{0}$, both known

$$
Q_{r}\left(X_{r}\right)=\frac{X_{r}-\mu_{0}}{\sigma_{0}}, r=1,2, \ldots
$$

2. Case UK: $\mu$ unknown, $\sigma=\sigma_{0}$ known

$Q_{r}\left(X_{r}\right)=\left(\frac{r-1}{r}\right)^{1 / 2} \frac{\left(X_{r}-\bar{X}_{r-1}\right)}{\sigma_{0}}, r=2,3, \ldots$

3. Case KU: $\mu=\mu_{0}$ known, $\sigma^{2}$ unknown

For this case, let

$$
S_{0, r}^{2}=\frac{1}{r} \sum_{j=1}^{r}\left(X_{j}-\mu_{0}\right)^{2} . \text { Thus, }
$$

$Q_{r}\left(X_{r}\right)=\Phi^{-1}\left\{G_{r-1}\left(\frac{X_{r}-\mu_{0}}{S_{0, r-1}}\right)\right\}, r=2,3, \ldots$ 
4. Case UU: $\mu$ and $\sigma^{2}$ both unknown

$$
\begin{gathered}
Q_{r}\left(X_{r}\right) \\
=\Phi^{-1}\left\{G_{r-2}\left[\left(\frac{r-1}{r}\right)^{1 / 2}\left(\frac{X_{r}-\bar{X}_{r-1}}{S_{r-1}}\right)\right]\right\} \\
r=3,4, \ldots
\end{gathered}
$$

where $S_{r}^{2}=\frac{1}{r-1} \sum_{j=1}^{r}\left(X_{j}-\bar{X}_{r}\right)^{2}$.

Note that in eqs. (1) - (4) above, $\bar{X}_{r}$ represents the sample mean estimated from the first $r$ observations, i.e., $\bar{X}_{r}=\frac{\sum_{i=1}^{r} X_{i}}{r}$.

Table 1. Notations for distribution functions.

$\Phi^{-1}(\cdot)$ - The inverse of the standard normal distribution function.

$G_{v}(\cdot)$ - The student- $t$ distribution function with $v$ degrees of freedom.

Q Chart Incorporating A Robust Estimator Of Scale

The $Q$ chart statistics in eqs. (1) and (2) are based on known variance while that of eqs. (3) and (4) are based on the estimated variance. A simulation study performed by Quesenberry (1995a) shows that the performance of a $Q$ chart for cases KK and UK are superior to that of cases KU and UU. In this paper, a method to improve the performance of a basic $Q$ chart using a robust estimator of scale will be suggested. The robust estimator of scale is based on a modified mean square successive difference (MSSD) approach.

Holmes and Mergen (1993) provide some discussion on this approach. Let the new estimator of the process dispersion be denoted by $S_{\text {MSSD }}$ while the new $Q$ statistics be represented by $Q_{\mathrm{MSSD}}$. The standard normal
$Q_{\text {MSSD }}$ statistics are shown below for cases $\mathrm{KU}$ and UU. All the notations which are used here are similar to that defined in Table 1 . Let $X_{1}, X_{2}, \ldots$ represent measurements made on a sequence of parts as they are produced in time and assume that these values are independently and identically distributed (i.i.d.) with a normal, $N\left(\mu, \sigma^{2}\right)$ process distribution. The two cases are:

1. Case KU: $\mu=\mu_{0}$ known, $\sigma^{2}$ unknown For odd numbered observations, i.e., when $r$ is an odd number,

$Q_{\mathrm{MSSD}, r}\left(X_{r}\right)$
$=\Phi^{-1}\left\{G_{\frac{r-1}{2}}\left[\frac{\sqrt{2}\left(X_{r}-\mu_{0}\right)}{S_{\mathrm{MSSD}, r-1}}\right]\right\}, r=3,5, \ldots$

where $S_{\mathrm{MSSD}, r-1}^{2}=\frac{2}{r-1} \sum_{i=2,4,6}^{r-1}\left(X_{i}-X_{i-1}\right)^{2}$.

For even numbered observations, i.e., when $r$ is an even number,

$Q_{\mathrm{MSSD}, r}\left(X_{r}\right)$
$=\Phi^{-1}\left\{G_{\frac{r-2}{2}}\left[\frac{\sqrt{2}\left(X_{r}-\mu_{0}\right)}{S_{\mathrm{MSSD}, r-2}}\right]\right\}, r=4,6, \ldots$

where $S_{\mathrm{MSSD}, r-2}^{2}=\frac{2}{r-2} \sum_{i=2,4,6}^{r-2}\left(X_{i}-X_{i-1}\right)^{2}$.

2. Case UU: $\mu$ and $\sigma^{2}$ both unknown

For this case, let $\bar{X}_{r}=\frac{\sum_{i=1}^{r} X_{i}}{r}$. For odd numbered observations, i.e., when $r$ is an odd number, 


$$
\begin{aligned}
& Q_{\mathrm{MSSD}, r}\left(X_{r}\right) \\
& =\Phi^{-1}\left\{G_{\frac{r-1}{2}}\left[\frac{\sqrt{\frac{2(r-1)}{r}}\left(X_{r}-\bar{X}_{r-1}\right)}{S_{\mathrm{MSSD}, r-1}}\right]\right\}, r=3,5, \ldots
\end{aligned}
$$

where $S_{\mathrm{MSSD}, r-1}^{2}=\frac{2}{r-1} \sum_{i=2,4,6}^{r-1}\left(X_{i}-X_{i-1}\right)^{2}$.

For even numbered observations, i.e., when $r$ is an even number,

$$
\begin{aligned}
& Q_{\mathrm{MSSD}, r}\left(X_{r}\right) \\
& =\Phi^{-1}\left\{G_{\frac{r-2}{2}}\left[\frac{\sqrt{\frac{2(r-1)}{r}}\left(X_{r}-\bar{X}_{r-1}\right)}{S_{\mathrm{MSSD}, r-2}}\right]\right\}, r=4,6, \ldots
\end{aligned}
$$

where $S_{\mathrm{MSSD}, r-2}^{2}=\frac{2}{r-2} \sum_{i=2,4,6}^{r-2}\left(X_{i}-X_{i-1}\right)^{2}$.

Note that the $Q_{\text {MSsD }}$ statistics in Eqs. (5a), (5b), (6a) and (6b) are standard normal random variables.

Tests For Shifts In The Process Mean

To enable a comparison in the performances of the proposed $Q_{\mathrm{MSSD}}$ chart and the basic $Q$ chart to be made in the next section, the following tests which are used by Quesenberry (1995a) will be considered: Given a sequence of say, $Q$ statistics, $Q_{t}, Q_{t-1}, \ldots$, these tests are defined as follow:

The 1-of-1 test: When $Q_{t}$ is plotted, the test signals an increase in $\mu$ if $Q_{t}>3$, and signals a decrease in $\mu$ if $Q_{t}<-3$.

The 9-of-9 test: When $Q_{t}$ is plotted, the test signals an increase in $\mu$ if $Q_{t}, Q_{t-1}, \ldots, Q_{t-8}$ all exceed 0 , and a decrease in $\mu$ if $Q_{t}, Q_{t-1}, \ldots, Q_{t-8}$ are all less than 0 . This test can only be employed if nine consecutive $Q$ statistics are available.

The 3-of-3 test: When $Q_{t}$ is plotted, the test signals an increase in $\mu$ if $Q_{t}, Q_{t-1}$ and $Q_{t-2}$ all exceed 1 , and a decrease in $\mu$ if $Q_{t}, Q_{t-1}$ and
$Q_{t-2}$ are all less than -1 . This test can only be employed if three consecutive $Q$ statistics are available.

The 4-of-5 test: When $Q_{t}$ is plotted, the test signals an increase in $\mu$ if at least four of the five values $Q_{t}, Q_{t-1}, \ldots, Q_{t-4}$ exceed 1 , and a decrease in $\mu$ if at least four of the five values $Q_{t}, Q_{t-1}, \ldots, Q_{t-4}$ are less than -1 . This test can only be employed if five consecutive $Q$ statistics are available.

The EWMA test: The EWMA statistic $Z_{t}$ is given by

$$
Z_{t}=\lambda Q_{t}+(1-\lambda) Z_{t-1}, \quad t=1,2, \ldots
$$

with $Z_{0}=0$. The $Z_{t}, t=1,2, \ldots$, values are plotted on a chart with limits at $\pm K \sqrt{\lambda /(2-\lambda)}$. The same values of $(\lambda, K)=(0.25,2.90)$ considered by Quesenberry (1995a) which gives control limits at \pm 1.096 are used in the next section. These limits give an in-control ARL of 372.6. If $Z_{t}>1.096$, an increase in $\mu$ is signaled and if $Z_{t}<-1.096$, a decrease in $\mu$ is signaled. The CUSUM test: The CUSUM statistics are defined as follow:

and

$$
S_{t}^{+}=\max \left\{0, S_{t-1}^{+}+Q_{t}-k_{s}\right\}
$$

$$
S_{t}^{-}=\min \left\{0, S_{t-1}^{-}+Q_{t}+k_{s}\right\}
$$

where $S_{0}^{+}=S_{0}^{-}=0$. An increase in $\mu$ is detected if $S_{t}^{+}>h_{s}$ and a decrease in $\mu$ if $S_{t}^{-}<-h_{s}$. Similar to Quesenberry (1995a), the values of $k_{s}=0.75$ and $h_{s}=3.34$ are used in this study. These values of $k_{s}$ and $h_{s}$ give an in-control ARL of 370.5 .

Evaluating The Performance Of The $Q_{\text {Msss }}$ Chart

A Monte Carlo simulation study is conducted using SAS version 8 to study the performance of the $Q_{\mathrm{MSSD}}$ chart based on cases $\mathrm{KU}$ and UU. Let the in-control mean be $\mu_{0}$ and the o.o.c. mean be $\mu_{s}=\mu_{0}+\delta \sigma$. Here, $\mu$ shifts 
from $\mu_{0}$ to $\mu_{s}$ after $r=c$, where $c \in\{5,20$, $100\}$ and $\delta \in\{0,0.5,1,1.5,2,3,4,5,6\} . c$ observations are generated from a $N(0,1)$ distribution and then 30 additional observations from a $N(\delta, 1)$ distribution. The $Q_{\mathrm{MSSD}}$ statistics in Eqs. (5a), (5b), (6a) and (6b) are computed as soon as enough values are available to define the particular statistic. This procedure is repeated 5000 times and the proportions of times a signal is observed for the $Q_{\mathrm{MSSD}}$ chart from $c+1$ to $c$ +30 , for the first time are recorded and are given in Tables 2, 3 and 4 for $c=5,20$ and 100 respectively. Note that this simulation study is conducted under the same condition as that in Quesenberry (1995a) so that a comparison between the performances of the $Q_{\mathrm{MSSD}}$ chart and the basic $Q$ chart can be made.

All the six tests discussed in the previous section are used in the simulation study. The results of the six tests for the basic $Q$ chart are obtained from Quesenberry (1995a). Since we are interested to detect positive shifts in the process mean, only the upper sided tests are considered. Here, an o.o.c. is signaled if the chart's statistics plot above the upper control limit of the test.

The results in Tables 2, 3 and 4 show that the proportions of o.o.c. for the 1-of-1 test when $\delta=0$ for both the $Q_{\mathrm{MSSD}}$ and $Q$ charts are about the same, thus the two charts have almost similar Type-I errors. For the 1-of-1 test where $\delta$
$>0$, the $Q_{\mathrm{MSSD}}$ chart has higher o.o.c. proportions compared to the $Q$ chart. Therefore, the new approach based on the 1-of-1 test has increased the sensitivity of the basic $Q$ chart while maintaining the same rate of Type-I error. On the whole, the Type-I errors of the 9-of-9, 3of-3, 4-of-5, EWMA and CUSUM tests for $c=5$ and 20 are higher for the $Q_{\text {MSSD }}$ chart compared to the $Q$ chart. However, for $c=100$, these tests produce similar Type-I errors for both the $Q_{\mathrm{MSSD}}$ and $Q$ charts.

The percentage of an increase in the sensitivity of the $Q_{\mathrm{MSSD}}$ chart using these tests in detecting shifts in the mean is greater than its increase in the Type- I error in comparison to the $Q$ chart. For example, in Table 2, using the 3-of3 test for case UU with $\delta=0.5$, an increase of about 2.7 fold in the sensitivity of the $Q_{\text {MSSD }}$ chart over the $Q$ chart is recorded. For this case, the false alarm rate of the $Q_{\mathrm{MSSD}}$ chart increase by only about 1.9 fold in comparison to that of the $Q$ chart. The results in Tables 2, 3 and 4 clearly indicate that the $Q_{\mathrm{MSSD}}$ chart is superior to the $\mathrm{Q}$ chart.

It should be noted that case UU has lower o.o.c. proportions than case KU for $\delta>0$ irrespective of the test that is used. The $Q_{\text {MSSD }}$ and $Q$ charts become more sensitive to process shifts as the value of $c$ increases. 
Table 2. Proportions of signaling an out-of-control for the $Q$ and $Q_{\mathrm{MSSD}}$ charts based on $c=5$.

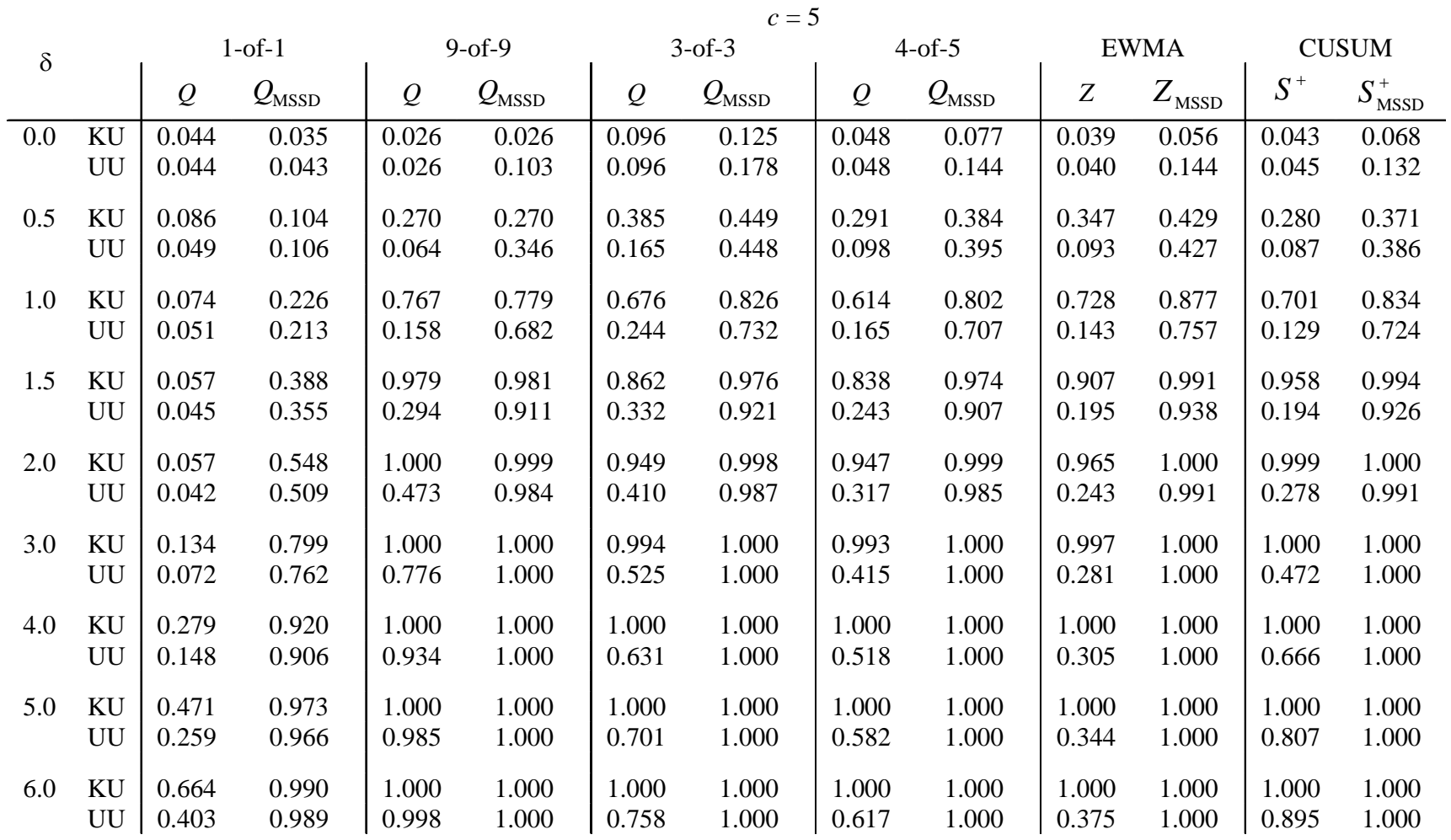

Table 3. Proportions of signaling an out-of-control for the $Q$ and $Q_{\mathrm{MSSD}}$ charts based on $c=20$.

\begin{tabular}{|c|c|c|c|c|c|c|c|c|c|c|c|c|c|}
\hline \multirow[b]{2}{*}{$\delta$} & & \multicolumn{12}{|c|}{$c=20$} \\
\hline & & $Q$ & $Q_{\mathrm{MSSD}}$ & $Q$ & $Q_{\mathrm{MSSD}}$ & $Q$ & $Q_{\mathrm{MSSD}}$ & $Q$ & $Q_{\mathrm{MSSD}}$ & $Z$ & $Z_{\mathrm{MSSD}}$ & $S^{+}$ & $S_{\mathrm{MSSD}}^{+}$ \\
\hline \multirow[t]{2}{*}{0.0} & KU & 0.044 & 0.035 & 0.030 & 0.029 & 0.095 & 0.113 & 0.047 & 0.065 & 0.041 & 0.048 & 0.042 & 0.056 \\
\hline & UU & 0.045 & 0.037 & 0.033 & 0.046 & 0.091 & 0.131 & 0.052 & 0.089 & 0.038 & 0.083 & 0.042 & 0.081 \\
\hline \multirow[t]{2}{*}{0.5} & KU & 0.116 & 0.125 & 0.270 & 0.271 & 0.425 & 0.455 & 0.333 & 0.376 & 0.421 & 0.447 & 0.360 & 0.400 \\
\hline & UU & 0.083 & 0.128 & 0.140 & 0.304 & 0.278 & 0.450 & 0.191 & 0.377 & 0.210 & 0.446 & 0.183 & 0.399 \\
\hline \multirow[t]{2}{*}{1.0} & KU & 0.175 & 0.330 & 0.774 & 0.777 & 0.798 & 0.873 & 0.746 & 0.857 & 0.906 & 0.936 & 0.888 & 0.913 \\
\hline & UU & 0.119 & 0.320 & 0.412 & 0.748 & 0.526 & 0.828 & 0.424 & 0.805 & 0.538 & 0.879 & 0.493 & 0.853 \\
\hline \multirow[t]{2}{*}{1.5} & KU & 0.237 & 0.609 & 0.981 & 0.978 & 0.970 & 0.993 & 0.967 & 0.994 & 0.997 & 1.000 & 1.000 & 0.999 \\
\hline & UU & 0.172 & 0.579 & 0.717 & 0.962 & 0.751 & 0.981 & 0.709 & 0.980 & 0.838 & 0.993 & 0.848 & 0.993 \\
\hline \multirow[t]{2}{*}{2.0} & KU & 0.334 & 0.846 & 0.999 & 0.999 & 0.997 & 1.000 & 0.998 & 1.000 & 1.000 & 1.000 & 1.000 & 1.000 \\
\hline & UU & 0.253 & 0.811 & 0.919 & 0.997 & 0.915 & 0.999 & 0.903 & 0.999 & 0.969 & 1.000 & 0.986 & 1.000 \\
\hline \multirow[t]{2}{*}{3.0} & KU & 0.623 & 0.993 & 1.000 & 1.000 & 1.000 & 1.000 & 1.000 & 1.000 & 1.000 & 1.000 & 1.000 & 1.000 \\
\hline & UU & 0.516 & 0.989 & 0.997 & 1.000 & 0.994 & 1.000 & 0.993 & 1.000 & 0.999 & 1.000 & 1.000 & 1.000 \\
\hline \multirow[t]{2}{*}{4.0} & KU & 0.887 & 1.000 & 1.000 & 1.000 & 1.000 & 1.000 & 1.000 & 1.000 & 1.000 & 1.000 & 1.000 & 1.000 \\
\hline & UU & 0.816 & 1.000 & 1.000 & 1.000 & 1.000 & 1.000 & 1.000 & 1.000 & 1.000 & 1.000 & 1.000 & 1.000 \\
\hline \multirow[t]{2}{*}{5.0} & KU & 0.986 & 1.000 & 1.000 & 1.00 & 1.000 & 1.000 & 1.000 & 1.000 & 1.000 & 1.000 & 1.000 & 1.000 \\
\hline & UU & 0.962 & 1.000 & 1.000 & 1.000 & 1.000 & 1.000 & 1.000 & 1.000 & 1.000 & 1.000 & 1.000 & 1.000 \\
\hline \multirow[t]{2}{*}{6.0} & KU & 1.000 & 1.0 & 1.00 & 1.0 & 1.000 & 1.000 & 1.000 & $1 .($ & 1.000 & 1.0 & 1.000 & 1.000 \\
\hline & UU & 0.997 & 1.000 & 1.000 & 1.000 & 1.000 & 1.000 & 1.000 & 1.000 & 1.000 & 1.000 & 1.000 & 1.000 \\
\hline
\end{tabular}


Table 4. Proportions of signaling an out-of-control for the $Q$ and $Q_{\mathrm{MSSD}}$ charts based on $c=100$.

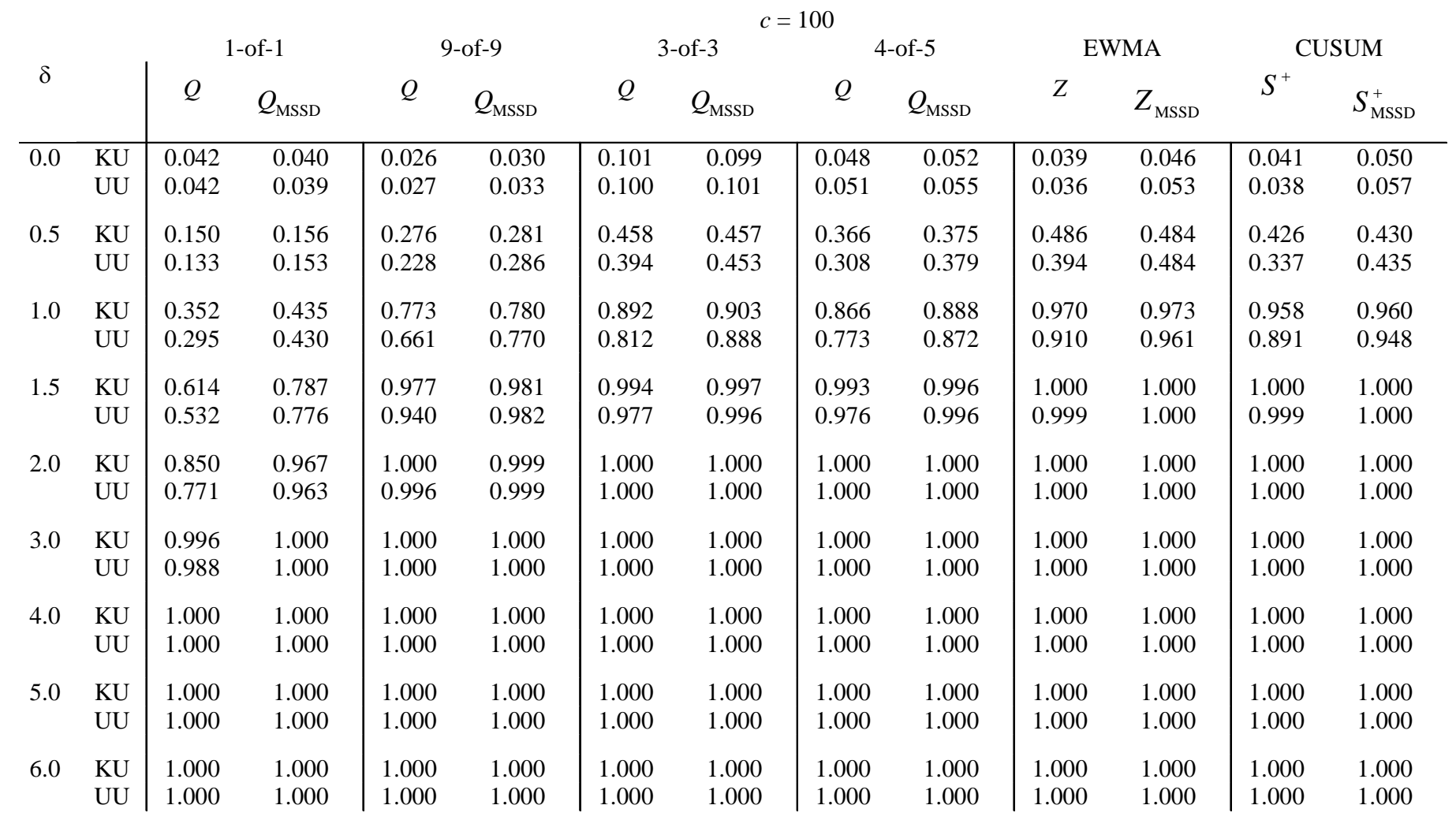

An Example of Application

This example is based on simulated data. Observations are generated using SAS version 8. The first 10 observations are generated from a standard normal, $N(0,1)$ distribution followed by 20 additional observations from a $N(1,1)$ distribution. The first 10 observations represent the in-control (stable) process while the next 20 the out-of-control (o.o.c.) process. The o.o.c. process involves a shift of one standard deviation in the mean. The simulated data and the corresponding computed statistics of $Q_{r}\left(X_{r}\right), Z_{r}, S_{r}^{+}, Q_{\mathrm{MSSD}, \mathrm{r}}\left(X_{r}\right)$, $Z_{\mathrm{MSSD}, r}$ and $S_{\mathrm{MSSD}, r}^{+}$are given in Table 5. The control charts plotted from the $Q$, EWMA (Z) and CUSUM $\left(S^{+}\right)$statistics are shown in Figure 1. Figure 2 gives the control charts plotted from the $Q_{\mathrm{MSSD}}$, EWMA $\left(Z_{\mathrm{MSSD}}\right)$ and CUSUM $\left(S_{\text {MSSD }}^{+}\right)$statistics. All the six tests which are considered in the simulation study will be used here. Because a positive shift is simulated, only the upper limits of each of the tests are used. The upper limits of 1.096 and 3.34 for the EWMA and CUSUM tests respectively are used. These upper limits correspond to an in-control ARL of approximately 370 for the case of the two-sided charts.

An o.o.c. signal is given by the 3-of-3 test at observation 25 in Figure 1. Note that the other tests fail to detect an o.o.c. in Figure 1. In Figure 2, o.o.c. signals are detected for the first time at observation 17 by the 3-of-3 test and at observation 19 by the 4-of-5 and 9-of-9 tests. Here, the EWMA ( $Z_{\mathrm{MSSD}}$ ) and CUSUM ( $S_{\text {MSSD }}^{+}$) tests issue o.o.c. signals for the first time at observations 25 and 26 respectively. This example shows that the $Q_{\text {MSsD }}$ chart is more sensitive to shifts compared to the $Q$ chart proposed by Quesenberry (1991 \& 1995a). 
Table 5. Simulated data and the computed statistics for 30 observations.

\begin{tabular}{|c|c|c|c|c|c|c|c|}
\hline $\begin{array}{l}\text { Obs. } \\
\text { No., } r\end{array}$ & $X_{r}$ & $Q_{r}\left(X_{r}\right)$ & $\begin{array}{c}\text { EWMA } \\
\left(Z_{r}\right)\end{array}$ & $\begin{array}{c}\text { CUSUM } \\
\left(S_{r}^{+}\right)\end{array}$ & $Q_{\mathrm{MSSD}, r}\left(X_{r}\right)$ & $\begin{array}{l}\text { EWMA } \\
\left(Z_{\mathrm{MSSD}, r}\right)\end{array}$ & $\begin{array}{l}\text { CUSUM } \\
\left(S_{\mathrm{MSSD}, r}^{+}\right)\end{array}$ \\
\hline 1 & -0.862 & - & - & - & - & - & - \\
\hline 2 & 2.519 & - & - & - & - & - & - \\
\hline 3 & -1.350 & -0.700 & -0.175 & 0 & -0.535 & -0.134 & 0 \\
\hline 4 & -0.332 & -0.346 & -0.218 & 0 & -0.125 & -0.132 & 0 \\
\hline 5 & 0.228 & 0.239 & -0.104 & 0 & 0.105 & -0.072 & 0 \\
\hline 6 & -1.499 & -1.571 & -0.470 & 0 & -0.660 & -0.219 & 0 \\
\hline 7 & 0.312 & 0.515 & -0.224 & 0 & 0.278 & -0.095 & 0 \\
\hline 8 & 0.384 & 0.556 & -0.029 & 0 & 0.280 & -0.001 & 0 \\
\hline 9 & -0.162 & -0.100 & -0.047 & 0 & -0.056 & -0.015 & 0 \\
\hline 10 & -2.233 & -2.232 & -0.593 & 0 & -1.236 & -0.320 & 0 \\
\hline 11 & 0.972 & 1.160 & -0.155 & 0.410 & 0.793 & -0.042 & 0.043 \\
\hline 12 & 2.524 & 2.209 & 0.436 & 1.869 & 1.588 & 0.366 & 0.881 \\
\hline 13 & 0.350 & 0.234 & 0.386 & 1.353 & 0.437 & 0.383 & 0.568 \\
\hline 14 & 0.457 & 0.311 & 0.367 & 0.914 & 0.509 & 0.415 & 0.327 \\
\hline 15 & 1.206 & 0.906 & 0.502 & 1.071 & 1.068 & 0.578 & 0.645 \\
\hline 16 & 1.845 & 1.354 & 0.715 & 1.675 & 1.466 & 0.800 & 1.360 \\
\hline 17 & 2.349 & 1.608 & 0.938 & 2.533 & 1.863 & 1.066 & 2.473 \\
\hline 18 & 0.301 & -0.072 & 0.686 & 1.711 & 0.473 & 0.918 & 2.196 \\
\hline 19 & 1.317 & 0.730 & 0.697 & 1.691 & 1.203 & 0.989 & 2.649 \\
\hline 20 & 0.148 & -0.234 & 0.464 & 0.707 & 0.346 & 0.828 & 2.246 \\
\hline 21 & 0.638 & 0.177 & 0.392 & 0.135 & 0.742 & 0.807 & 2.238 \\
\hline 22 & -1.656 & -1.709 & -0.133 & 0 & -1.058 & 0.340 & 0.429 \\
\hline 23 & 1.640 & 1.038 & 0.160 & 0.288 & 1.433 & 0.614 & 1.112 \\
\hline 24 & 2.245 & 1.457 & 0.484 & 0.996 & 1.824 & 0.916 & 2.186 \\
\hline 25 & 1.871 & 1.086 & 0.635 & 1.332 & 1.654 & 1.100 & 3.090 \\
\hline 26 & 1.390 & 0.672 & 0.644 & 1.254 & 1.317 & 1.155 & 3.657 \\
\hline 27 & 1.690 & 0.888 & 0.705 & 1.392 & 1.592 & 1.264 & 4.498 \\
\hline 28 & 3.085 & 1.909 & 1.006 & 2.551 & 2.505 & 1.574 & 6.253 \\
\hline 29 & 0.717 & 0.019 & 0.759 & 1.820 & 0.855 & 1.394 & 6.358 \\
\hline 30 & 1.278 & 0.451 & 0.682 & 1.521 & 1.303 & 1.372 & 6.912 \\
\hline
\end{tabular}



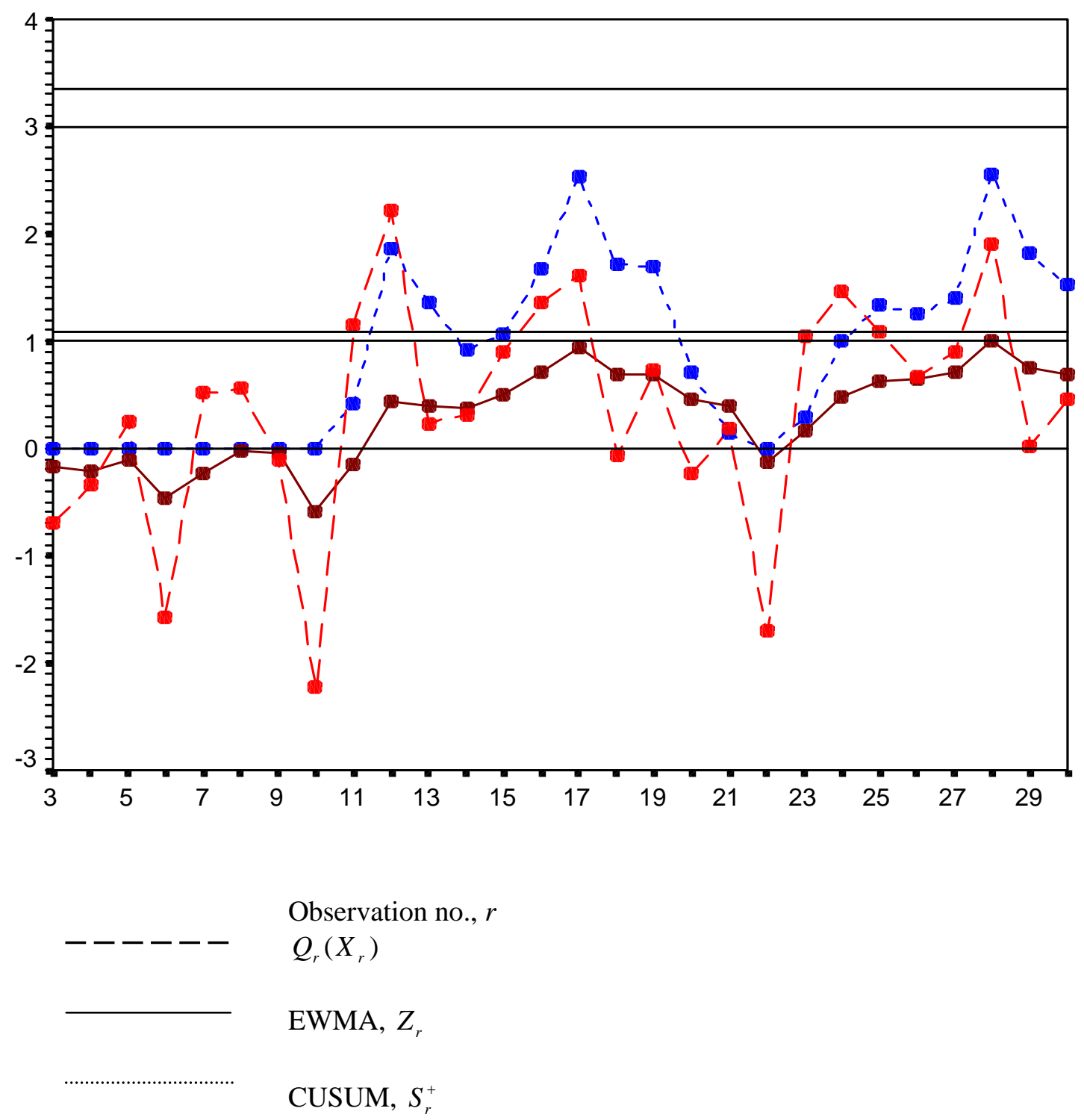

Figure 1. The charts constructed from the $Q$, EWMA $(Z)$ and CUSUM $\left(S^{+}\right)$statistics in Table 5. 


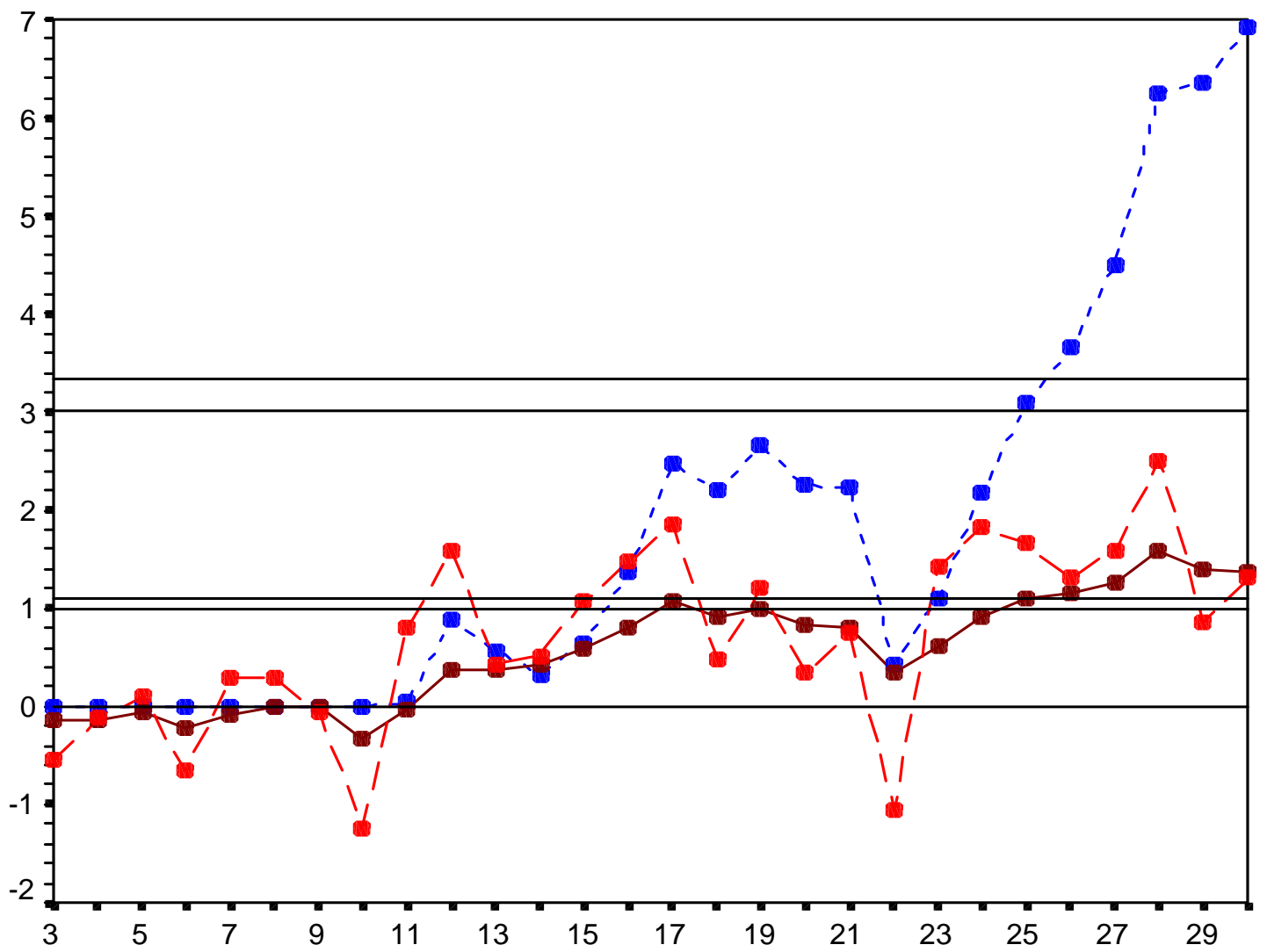

Observation no., $r$

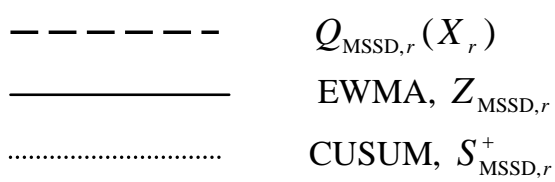

Figure 2. The charts constructed from the $Q_{\mathrm{MSSD}}$, EWMA ( $\left.Z_{\mathrm{MSSD}}\right)$ and CUSUM ( $\left.S_{\mathrm{MSSD}}^{+}\right)$statistics in Table 5. 


\section{Conclusion}

This article demonstrates that the performance of the basic $Q$ chart for cases KU and UU have improved tremendously by incorporating a robust estimator of scale based on a modified mean square successive difference approach. The proofs of how the $Q_{\text {MSSD }}$ statistics in eqs. (5a), (5b), (6a) and (6b) which are i.i.d. standard normal random variables are derived will be given in the Appendix.

\section{References}

Holmes, D. S., \& Mergen, A. E. (1993). Improving the performance of the $T^{2}$ control chart. Quality Engineering, 5, 619 - 625.
Mood, A. M., Graybill, F. A., \& Boes, D. C. (1974). Introduction to the theory of statistics. New York: McGraw-Hill.

Quesenberry, C. P. (1991). SPC Q charts for start-up processes and short or long runs. Journal of Quality Technology, 23, 213 224.

Quesenberry, C. P. (1995a). On properties of Q charts for variables. Journal of Quality Technology, 27, 184 - 203.

Quesenberry, C. P. (1995b). On properties of binomial Q charts for attributes. Journal of Quality Technology, 27, 204 - 213.

Quesenberry, C. P. (1995c). On properties of Poisson Q charts for attributes. Journal of Quality Technology, 27, 293 - 303.

Quesenberry, C. P. (1995d). Geometric Q charts for high quality processes. Journal of Quality Technology, 27, 304 - 315.

Appendix

Notation used here were defined above. The following theorem (Mood, Graybill \& Boes, 1974) is required.

Theorem:

If $Z$ has a standard normal distribution and $V$ has a chi-square distribution with $k$ degrees of freedom, and $Z$ and $V$ are independent, then

$$
T=\frac{Z}{\sqrt{V / k}}(9)
$$

has a student- $t$ distribution with $k$ degrees of freedom.

\section{Equation 5(a): Case KU}

For odd numbered observations, i.e., when $r$ is an odd number,

$$
Q_{\mathrm{MSSD}, r}\left(X_{r}\right)=\Phi^{-1}\left\{G_{\frac{r-1}{2}}\left[\frac{\sqrt{2}\left(X_{r}-\mu_{0}\right)}{S_{\mathrm{MSSD}, r-1}}\right]\right\} \sim N(0,1), r=3,5, \ldots
$$


Proof:

If $X_{j}, j=1,2, \ldots$, are i.i.d. $N\left(\mu_{0}, \sigma^{2}\right)$ variables, then $X_{i}-X_{i-1} \sim N\left(0,2 \sigma^{2}\right), i=2,4, \ldots$

and $\quad \frac{1}{\sigma \sqrt{2}}\left(X_{i}-X_{i-1}\right) \sim N(0,1), i=2,4, \ldots$ Thus, $\quad \frac{1}{2 \sigma^{2}}\left(X_{i}-X_{i-1}\right)^{2} \sim \chi_{1}^{2}$

and $\quad \frac{1}{2 \sigma^{2}} \sum_{i=2,4,6}^{r-1}\left(X_{i}-X_{i-1}\right)^{2} \sim \chi_{\frac{r-1}{2}}^{2} . \quad$ Let $S_{\mathrm{MSSD}, r-1}^{2}=\frac{2}{r-1} \sum_{i=2,4,6}^{r-1}\left(X_{i}-X_{i-1}\right)^{2}$

then, $\quad S_{\mathrm{MSSD}, r-1}^{2} \sim \frac{4 \sigma^{2}}{r-1} \chi_{\frac{r-1}{2}}^{2}$. Because $\frac{1}{\sigma}\left(X_{r}-\mu_{0}\right) \sim N(0,1)$

then, from Eq. (9) in the above theorem, $\frac{\frac{1}{\sigma}\left(X_{r}-\mu_{0}\right)}{\sqrt{\left(\frac{r-1}{4 \sigma^{2}}\right) S_{\mathrm{MSSD}, r-1}^{2} /\left(\frac{r-1}{2}\right)}} \sim t_{\frac{r-1}{2}}$,

i.e., $\quad \frac{\sqrt{2}\left(X_{r}-\mu_{0}\right)}{S_{\mathrm{MSSD}, r-1}} \sim t_{\frac{r-1}{2}}$. Thus, $Q_{\mathrm{MSSD}, r}\left(X_{r}\right)=\Phi^{-1}\left\{G_{\frac{r-1}{2}}\left[\frac{\sqrt{2}\left(X_{r}-\mu_{0}\right)}{S_{\mathrm{MSSD}, r-1}}\right]\right\} \sim N(0,1), r=3,5, \ldots$.

Equation 5(b): Case KU

It must be shown that for even numbered observations, i.e., when $r$ is an even number,

$$
Q_{\mathrm{MSSD}, r}\left(X_{r}\right)=\Phi^{-1}\left\{G_{\frac{r-2}{2}}\left[\frac{\sqrt{2}\left(X_{r}-\mu_{0}\right)}{S_{\mathrm{MSSD}, r-2}}\right]\right\} \sim N(0,1), r=4,6, \ldots
$$

Proof:

If $X_{j}, j=1,2, \ldots$, are i.i.d. $N\left(\mu_{0}, \sigma^{2}\right)$ variables, then $X_{i}-X_{i-1} \sim N\left(0,2 \sigma^{2}\right), i=2,4, \ldots$

and $\frac{1}{\sigma \sqrt{2}}\left(X_{i}-X_{i-1}\right) \sim N(0,1), i=2,4, \ldots$ Thus, $\frac{1}{2 \sigma^{2}}\left(X_{i}-X_{i-1}\right)^{2} \sim \chi_{1}^{2}$

and $\frac{1}{2 \sigma^{2}} \sum_{i=2,4,6}^{r-2}\left(X_{i}-X_{i-1}\right)^{2} \sim \chi_{\frac{r-2}{2}}^{2}$. Let $S_{\mathrm{MSSD}, r-2}^{2}=\frac{2}{r-2} \sum_{i=2,4,6}^{r-2}\left(X_{i}-X_{i-1}\right)^{2}$

then, $\quad S_{\mathrm{MSSD}, r-2}^{2} \sim \frac{4 \sigma^{2}}{r-2} \chi_{\frac{r-2}{2}}^{2}$.

Because $\frac{1}{\sigma}\left(X_{r}-\mu_{0}\right) \sim N(0,1)$, then, from Eq. (9) in the above theorem, $\frac{\frac{1}{\sigma}\left(X_{r}-\mu_{0}\right)}{\sqrt{\left(\frac{r-2}{4 \sigma^{2}}\right) S_{\mathrm{MSSD}, r-2}^{2} /\left(\frac{r-2}{2}\right)}} \sim$

$t_{\frac{r-2}{2}}$. i.e., $\frac{\sqrt{2}\left(X_{r}-\mu_{0}\right)}{S_{\mathrm{MSSD}, r-2}} \sim t_{\frac{r-2}{2}}$. Thus, $Q_{\mathrm{MSSD}, r}\left(X_{r}\right)=\Phi^{-1}\left\{G_{\frac{r-2}{2}}\left[\frac{\sqrt{2}\left(X_{r}-\mu_{0}\right)}{S_{\mathrm{MSSD}, r-2}}\right]\right\} \sim N(0,1), r=4,6, \ldots$. 
Equation 6(a): Case UU

It must be shown that for odd numbered observations, i.e., when $r$ is an odd number, $Q_{\mathrm{MSSD}, r}\left(X_{r}\right)=\Phi^{-1}\left\{G_{\frac{r-1}{2}}\left[\frac{\sqrt{\frac{2(r-1)}{r}}\left(X_{r}-\bar{X}_{r-1}\right)}{S_{\mathrm{MSSD}, r-1}}\right]\right\} \sim N(0,1), r=3,5, \ldots$

Proof:

If $X_{j}, j=1,2, \ldots$, are i.i.d. $N\left(\mu_{0}, \sigma^{2}\right)$ variables, then $\quad X_{i}-X_{i-1} \sim N\left(0,2 \sigma^{2}\right), \mathrm{i}=2,4, \ldots$

and

$\frac{1}{\sigma \sqrt{2}}\left(X_{i}-X_{i-1}\right) \sim N(0,1), i=2,4, \ldots \quad$ Thus, $\frac{1}{2 \sigma^{2}}\left(X_{i}-X_{i-1}\right)^{2} \sim \chi_{1}^{2}$

and $\frac{1}{2 \sigma^{2}} \sum_{i=2,4,6}^{r-1}\left(X_{i}-X_{i-1}\right)^{2} \sim \chi_{\frac{r-1}{2}}^{2}$.

Let $\quad S_{\mathrm{MSSD}, r-1}^{2}=\frac{2}{r-1} \sum_{i=2,4,6}^{r-1}\left(X_{i}-X_{i-1}\right)^{2}$ then, $\quad S_{\mathrm{MSSD}, r-1}^{2} \sim \frac{4 \sigma^{2}}{r-1} \chi_{\frac{r-1}{2}}^{2}$.

Because $\bar{X}_{r-1} \sim N\left(\mu_{0}, \frac{\sigma^{2}}{r-1}\right)$ then, $\frac{1}{\sigma} \sqrt{\frac{r-1}{r}}\left(X_{r}-\bar{X}_{r-1}\right) \sim N(0,1)$

from Eq. (9) in the above theorem, $\frac{\frac{1}{\sigma} \sqrt{\frac{r-1}{r}}\left(X_{r}-\bar{X}_{r-1}\right)}{\sqrt{\left(\frac{r-1}{4 \sigma^{2}}\right) S_{\text {MSSD },-1-1}^{2} /\left(\frac{r-1}{2}\right)}} \sim t_{\frac{r-1}{2}}$. i.e., $\frac{\sqrt{\frac{2(r-1)}{r}}\left(X_{r}-\bar{X}_{r-1}\right)}{S_{\text {MSSD }, r-1}} \sim t_{\frac{r-1}{2}}$.

Thus, $Q_{\mathrm{MSSD}, r}\left(X_{r}\right)=\Phi^{-1}\left\{G_{\frac{r-1}{2}}\left[\frac{\sqrt{\frac{2(r-1)}{r}}\left(X_{r}-\bar{X}_{r-1}\right)}{S_{\mathrm{MSSD}, r-1}}\right]\right\} \sim N(0,1), r=3,5, \ldots$

Equation 6(b): Case UU

It must be shown that for even numbered observations, i.e., when $r$ is an even number, $Q_{\mathrm{MSSD}, r}\left(X_{r}\right)=\Phi^{-1}\left\{G_{\frac{r-2}{2}}\left[\frac{\sqrt{\frac{2(r-1)}{r}}\left(X_{r}-\bar{X}_{r-1}\right)}{S_{\mathrm{MSSD}, r-2}}\right]\right\} \sim N(0,1), r=4,6, \ldots$

Proof:

If $X_{j}, j=1,2, \ldots$, are i.i.d. $N\left(\mu_{0}, \sigma^{2}\right)$ variables, then $X_{i}-X_{i-1} \sim N\left(0,2 \sigma^{2}\right), i=2,4, \ldots$

and $\frac{1}{\sigma \sqrt{2}}\left(X_{i}-X_{i-1}\right) \sim N(0,1), i=2,4, \ldots$ Thus, $\frac{1}{2 \sigma^{2}}\left(X_{i}-X_{i-1}\right)^{2} \sim \chi_{1}^{2}$

and $\frac{1}{2 \sigma^{2}} \sum_{i=2,4,6}^{r-2}\left(X_{i}-X_{i-1}\right)^{2} \sim \chi_{\frac{r-2}{2}}^{2}$.

Let $\quad S_{\mathrm{MSSD}, r-2}^{2}=\frac{2}{r-2} \sum_{i=2,4,6}^{r-2}\left(X_{i}-X_{i-1}\right)^{2}$ then, $\quad S_{\mathrm{MSSD}, r-2}^{2} \sim \frac{4 \sigma^{2}}{r-2} \chi_{\frac{r-2}{2}}^{2}$. 
Because $\bar{X}_{r-1} \sim N\left(\mu_{0}, \frac{\sigma^{2}}{r-1}\right)$ then, $\frac{1}{\sigma} \sqrt{\frac{r-1}{r}}\left(X_{r}-\bar{X}_{r-1}\right) \sim N(0,1)$

from Eq. (9) in the above theorem, $\frac{\frac{1}{\sigma} \sqrt{\frac{r-1}{r}}\left(X_{r}-\bar{X}_{r-1}\right)}{\sqrt{\left(\frac{r-2}{4 \sigma^{2}}\right) S_{\mathrm{MSSD}, r-2}^{2} /\left(\frac{r-2}{2}\right)}} \sim t_{\frac{r-2}{2}}$. i.e.,

$\frac{\sqrt{\frac{2(r-1)}{r}}\left(X_{r}-\bar{X}_{r-1}\right)}{S_{\mathrm{MSSD}, r-2}} \sim t_{\frac{r-2}{2}}$. Thus, $Q_{\mathrm{MSSD}, r}\left(X_{r}\right)=\Phi^{-1}\left\{G_{\frac{r-2}{2}}\left[\frac{\sqrt{\frac{2(r-1)}{r}}\left(X_{r}-\bar{X}_{r-1}\right)}{S_{\mathrm{MSSD}, r-2}}\right]\right\} \sim N(0,1), r=4,6, \ldots$ 